\title{
Carnosol, a dietary diterpene from rosemary (Rosmarinus officinalis) activates Nrf2 leading to sestrin 2 induction in colon cells
}

\author{
Miao Yan ${ }^{1,2}$, Bhaskar Vemu ${ }^{1}$, Jacob P Veenstra ${ }^{1}$, Sakina M Petiwala ${ }^{1}$ and Jeremy J Johnson ${ }^{1 *}$ \\ ${ }^{1}$ Department of Pharmacy Practice, College of Pharmacy, University of Illinois at Chicago, Chicago, Illinois, USA \\ ${ }^{2}$ Institute of Clinical Pharmacy, The Second Xiangya Hospital, Central South University, Changsha, Hunan Province, China
}

\begin{abstract}
Background: Rosemary is abundant with phytochemicals and has recently been approved as an antioxidant food preservative in the European Union. The safety of rosemary is well established, however, the benefits on gastrointestinal health are less known. Our overall hypothesis is that the phytochemicals in rosemary including carnosol have the potential to promote gastrointestinal health by activation of the antioxidant sestrin- 2 when consumed in our diet.
\end{abstract}

Methods: Colon cells HCT116 and SW480 were treated with carnosol and evaluated by MTT, immunofluorescence, ELISA, and Western blot analysis to understand the modulation of the PERK/Nrf2/Sestrin-2 pathway.

Results: Carnosol was found to modulate PERK and increase the concentration of nuclear Nrf2. Furthermore, a downstream marker of Nrf2 expression, Sestrin-2 was shown to be upregulated.

Conclusion: Based on these observations carnosol modulates the PERK and Nrf2 pathways along with increased expression of sestrin-2, a known stress inducible antioxidant.

\section{Introduction}

A significant amount of research from in vitro studies to epidemiological studies have reported on the benefits of the Mediterranean diet [i.e. low saturated fats, high poly unsaturated fatty acids, increased raw fruit and vegetable intake and herbs including rosemary (Rosmarinus officinalis)] in promoting overall health [1-3]. An underappreciated component of the Mediterranean diet in terms of health promotion includes herbs such as rosemary, basil, oregano, and sage [4]. The Mediterranean diet is known to be rich in phytochemicals with positive outcomes on research models in cardiovascular function, diabetes, inflammation, and cancer $[5,6]$. Rosemary extracts standardized to the diterpenes carnosic acid and carnosol have been approved in the European Union (EU) as an antioxidant food preservative and in the United States as a GRAS (Generally Recognized as Safe) status as a food ingredient [4,7]. The anticancer properties of rosemary have been suggested to be attributed to its major polyphenolic constituents, carnosic acid and carnosol [8]. Within rosemary there are many phytochemicals, however, carnosic acid and carnosol have been reported to contribute to $90 \%$ of the antioxidant activity [9]. Carnosic acid is the most abundant diterpenes and is readily oxidized to carnosol and represents a possible active metabolite of carnosic acid [10]. Carnosol has been shown to have many pharmacological properties that could be beneficial for modulating deregulated cell signaling pathways in cancer cells $[3,11,13]$.

A growing body of evidence indicates that oxidative stress is a critical component of disease etiology including cancer and inflammation [14]. In response to cellular stress the human body has the capability to offer a line of defense by innate antioxidant response network that is regulated by genes that have the antioxidant response elements (AREs) in the promoter region. One of the most studied AREs is in the NF-E2-relatedfactor-2 (Nrf2) gene which is a member of the cap'n' collar family of bZIP transcription factors $[15,16]$. Sestrin-2, the expression of which is responsive to hypoxia, DNA damage, and oxidative stress, may have cytoprotective activity against various stresses. A recent study suggested that the Nrf2/ARE pathway regulates sestrin-2 gene expression to combat various stresses including hypoxia, DNA damage, and oxidative stress [17]. Sestrin 2, also known as Hi95 is a member of the sestrin family linked to the tumor suppression p53. Interestingly, low expression of sestrin 2 has been associated with human colorectal cancers. Furthermore, low expression of Sestrin was associated with advanced tumor stage, lymphatic invasion, metastasis, vascular invasion, liver metastasis and decreased survival rate. From a clinical perspective, sestrin 2 deficiency was associated with decreased susceptibility to chemotherapeutic drugs.

${ }^{\star}$ Correspondence to: Jeremy J Johnson, PharmD, PhD, University of Illinois at Chicago, College of Pharmacy, Department of Pharmacy Practice, 833 South Wood Street, Chicago IL 60612-7230, USA, Tel: 3129964368, Fax: 312-996-0379; E-mail: jjjohn@uic.edu

Key words: carnosol, colon cancer, HCT116, SW480, Nrf2, sestrin, PERK, ER stress, UPR

Received: August 01, 2018; Accepted: August 09, 2018; Published: August 15, 2018 
Given that carnosic acid can be oxidized in biological systems to metabolites including carnosol we tested our hypothesis that the most abundant metabolite, carnosol is capable of inducing the Nrf2 network in colon cancer cells. More specifically, we evaluate the role of Nrf2 regulating the expression of sestrin 2 .

\section{Materials and methods}

\section{Carnosol, kits and antibodies}

Carnosol was obtained from Cayman Chemical Co. (Ann Arbor, MI. Lot: 127606-37, Purity $\geq 99 \%$ ). All antibodies for western blot analysis and cleaved caspase-3 ELISA kit were obtained from Cell Signaling Technology (Danvers, MA). DeadEnd ${ }^{\text {Tm }}$ Fluorometric TUNEL System was obtained from Promega (Madison, WI). BCA protein assay kit, chemiluminescent, NE-PER nuclear and cytoplasmic extraction reagents were obtained from Pierce (Rockford, IL). Nrf2 antibody (sc-722) for immunofluorescence was obtained from Santa Cruz Biotechnology, Inc. (Santa Cruz, CA). Fluorescein isothiocyanate (FITC)-conjugated goat-anti-rabbit antibody was obtained from Pierce (Rockford, IL). Antibodies purchased from Cell Signal Technology are listed with the catalog number in parentheses: Nrf2 (12721P), Erk (9911), Sestrin-2 (8487S), GPX1 (3206S), GSTP1 (3369S), SOD1 (2770S), PERK (5638P), p-PERK (3179S), and HO-1 (5853S). OneStep RT-PCR kit was obtained from Life Technologies (Grand Island, NY). RNeasy mini kit and RNase-Free DNase set for RNA extraction were obtained from QIAGEN (Santa Clarita, CA). TransIT-siQUEST transfection reagent was obtained from Mirus Bio LLC. Nrf2 siRNA(h) and Control siRNA-A were obtained from Santa Cruz Bio, Inc.

\section{Cell culture and treatment}

HCT116 and SW480 cells were obtained from American Type Culture Collection (Manassas, VA). HCT116 cells were cultured in McCoy's 5A with L-glutamine supplemented with $10 \%$ fetal bovine serum (FBS) and $1 \%$ penicillin/streptomycin. SW480 cells were cultured in MEM supplemented with 10\% FBS and 1\% penicillin/ streptomycin. Cells were cultured in medium supplemented with a range of concentrations of carnosol for desired times. Dimethyl sulfoxide (DMSO) was used to solubilize carnosol with the final DMSO concentration in the media $0.1 \%$. Vehicle controls were employed in all experiments.

\section{Cell viability}

Cell viability was determined by 3-(4, 5-dimethylthiazol-2-yl)-2, 5-diphenyltetrazolium bromide (MTT) assay as described previously [11]. The seeding densities of HCT116 and SW480 cells were both $2 \times 10^{4}$ in each well. Cells were cultured in medium supplemented with a range of concentrations of carnosol $(0,10,20,30,40,50,75$ and 100 $\mu \mathrm{M})$ for 24,48 or $72 \mathrm{~h}$.

\section{ELISA}

Cleaved Caspase-3 levels were detected with ELISA according to the manufacturer's protocol [12]. The seeding densities of HCT116 and SW480 cells were $4 \times 10^{6}$ and $8 \times 10^{6}$ respectively in each $10 \mathrm{~cm}^{2}$ plate. Cells were cultured in medium supplemented with carnosol $(0,40,50$ and $75 \mu \mathrm{M})$ for 24 or $48 \mathrm{~h}$.

\section{Immunofluorescence staining}

Cells were seeded in slides and incubated at $37^{\circ} \mathrm{C}$ for $24 \mathrm{~h}$. The cells were then incubated with $60 \mu \mathrm{M}$ carnosol for $24 \mathrm{~h}$, fixed with $4 \%$ paraformaldehyde, and carefully seeded on poly-L-lysine-coated coverslips. The cells were permeabilized with $0.3 \%$ Triton X-100, blocked with $3 \%$ bovine serum albumin, and incubated with sestrin-2 antibody (dilution of 1:200) overnight at $4^{\circ} \mathrm{C}$. The cells were then incubated with a fluorescein isothiocyanate (FITC)-conjugated goatanti-rabbit antibody, and the nuclei were stained with DAPI, and the slides were examined with a fluorescence microscope [13]. The seeding densities of HCT116 and SW480 cells were $2.5 \times 10^{5}$ and $5 \times 10^{5}$ respectively in each well. Cells were cultured in medium supplemented with carnosol $(60 \mu \mathrm{M})$ for $24 \mathrm{hr}$.

\section{Western blots}

Whole cell lysates from treated cells were prepared as previously described [13]. Lysates were quantified using BCA assay according to manufacturer's manual. Briefly, lysates were loaded to each well of $12 \%$ pre-cast gels (Bio-Rad, Hercules, CA). After transfer, membranes were blocked and incubated with primary antibody $(1: 1000)$ overnight at $4^{\circ} \mathrm{C}$, rinsed briefly, then incubated with secondary antibody (1:2000) at room temperature for $1 \mathrm{hr}$. Membranes were washed, incubated with substrates and exposed in a FluorChem E imager (ProteinSimple, Santa Clara, CA) $[11,13]$. The seeding densities of HCT116 and SW480 cells were $4 \times 10^{6}$ and $8 \times 10^{6}$ respectively in each $10 \mathrm{~cm}^{2}$ plate. Cells were cultured medium supplemented with a range of concentrations of carnosol $(0,40,50$ and $75 \mu \mathrm{M})$ for $24 \mathrm{~h}$. Western blots were performed with a minimum of three separate lysates. Separate lysates were then analyzed three times by western blot to confirm the results.

\section{Transfection with siRNA}

HCT116 or SW 480 cells were seeded in $10 \mathrm{~cm}^{2}$ plates and grown in culture medium. At 50-60\% confluence, the cells were transfected with desired small interfering RNA (siNrf2) targeting Nrf2 or ctrl-siRNA using TransIT-siQUEST transfection reagent in $1 \mathrm{~mL}$ of transfection medium. After $24 \mathrm{~h}$, the medium was replaced with fresh medium, and the cells were cultured for an additional $24 \mathrm{~h}$ before treating with a range of concentrations of carnosol for $24 \mathrm{~h}$. Then cell lysates were prepared, quantified and ready for western blots. The seeding densities of HCT 116 and SW 480 cells were $4 \times 10^{6}$ and $8 \times 10^{6}$, respectively, in each $10 \mathrm{~cm}^{2}$ plate.

\section{Statistical analysis}

All statistical analysis was performed by using VassarStats software. Data are expressed as mean with standard deviation for all groups. Statistical significance of differences in all measurements between control and treated groups was determined by one-way ANOVA followed by Tukey's HSD test for multiple comparisons. Student's paired $t$ test was used for pair wise group comparisons, as needed. All statistical tests were two-sided, and $\mathrm{P}<0.05$ was considered statistically significant.

\section{Results}

\section{Carnosol reduced cell viability and induced apoptosis on colon cancer cells}

Using an MTT assay we evaluated the impact of carnosol on cell viability and observed that carnosol decreased HCT116 and SW480 cell viability in a dose dependent manner (Figure 1A). DNA fragmentation was detected by TUNEL staining and revealed that carnosol promoted DNA fragmentation in HCT116 cells (Figure 1B). The effect was not as pronounced in SW480 cells which are known to have the oncogenes (e.g. myc, ras, fos) that have been associated with preventing apoptosis. Carnosol significantly increased cleaved caspase-3 at $75 \mu \mathrm{M}$ after 24 
hrs in HCT $116(\mathrm{p}<0.01)$ (Figure 1C). A slight increase was observed in SW480 $(\mathrm{p}<0.05)$ cells.

\section{Carnosol increased the expression of $\mathrm{Nrf} 2$ in colon cancer cells}

One model explaining the role of Nrf2 stipulates that, under normal conditions, it exists in an inactive, cytoplasm-localized state. Upon cellular stimulation by xenobiotics, electrophiles, or oxidativestress-generating agents, the cytoplasmic-retention mechanism is inactivated, and Nrf2 is transported to the nucleus, where it activates target-gene transcription $[16,18]$. Therefore, we examined if carnosol contributed to the activation of Nrf2. Both HCT 116 and SW480 cells were treated with increasing concentrations of carnosol and whole cell lysates were incubated with anti-Nrf2 antibody. Carnosol increased the accumulation of Nrf2 protein in both HCT116 and SW480 cells (Figure $2 \mathrm{~A}$ ) at $50 \mu \mathrm{M}$ and $40 \mu \mathrm{M}$ respectively. As reported previously by others, activation of Erk is required for Nrf2 nuclear localization and is upregulated following treatment with carnosol. Nrf2 is known to interact with the antioxidant response element (ARE) which has been shown to regulate hundreds of genes including sestrin-2, GPX1, GSTP1, and SOD1. A significant upregulation of sestrin-2 was observed in both cell lines. Interestingly, modest changes were observed in GPX1, GSTP1 and SOD1 in HCT116 while changes in SW480 were not observed.

\section{The accumulation of Nrf2 protein was also evaluated by} western blot

Activation of Nrf2 will result in translocation from the cytoplasm to the nucleus that will result in subsequent gene expression. To investigate whether carnosol treatment affects Nrf2 protein levels in the nuclear compartment, we extracted nuclear and cytoplasmic fractions from cells and performed immunoblot analysis. As shown in Figure $2 \mathrm{~B}$, carnosol significantly induced the accumulation of Nrf2 in the nuclear fraction with increases seen as early as $40 \mu \mathrm{M}$ and $50 \mu \mathrm{M}$ in HCT116 and SW480 cells, respectively. This can be further visualized by immunofluorescence after a 24 -hour treatment (Figure 2C).

\section{Carnosol modulate PERK/Nrf2/sestrin-2 pathway in HCT116 and SW480 cells}

Nrf2 has been shown to be a direct substrate of protein kinase (PKR)-like endoplasmic reticulum kinase (PERK) "and phosphorylated PERK is responsible for triggering the dissociation of the Nrf2/Keap1 complex and inhibits the re-association of Nrf2/Keap1 [19]. Previously, we have reported the modulation of the unfolded protein response pathway that includes PERK in prostate cancer cells using a rosemary extract rich in diterpenes including carnosol $[16,20]$. Given this line of reasoning it was plausible to investigate the potential activation of

[A]
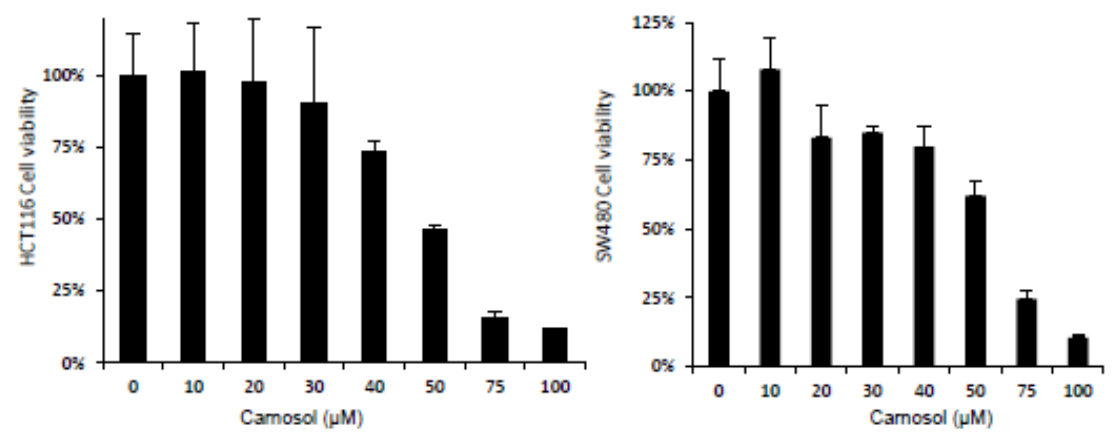

$[B]$
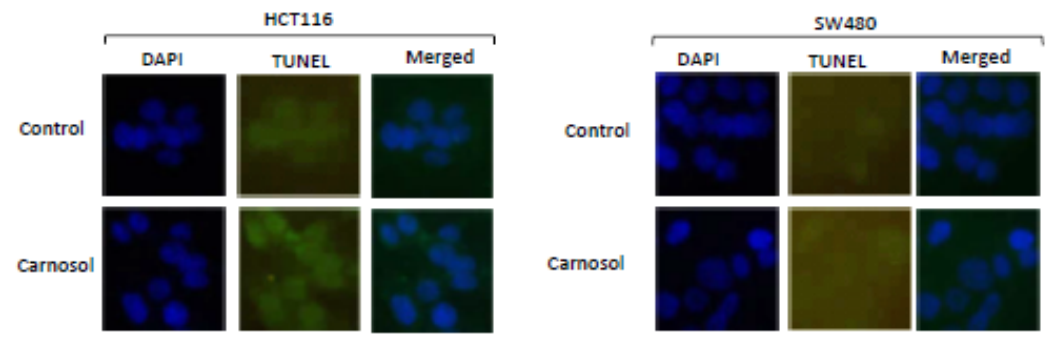

$[\mathrm{C}]$
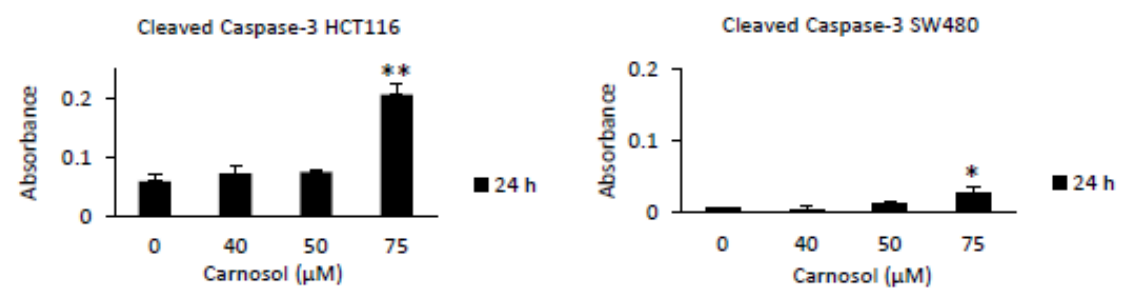

Figure 1. Carnosol decreased cell viability in HCT116 and SW480 colon cancer cells. (A) For cell viability evaluation, HCT116 and SW480 cells were treated with desired doses of carnosol for $72 \mathrm{hr}$ and then subjected to MTT assay. (B) Terminal deoxynucleotidyl transferase-mediated dUTP Nick-End Labeling (TUNEL) assay were performed to visualize cell apoptosis in treated colon cells. (C) For cleaved caspase-3 ELISA, cells were treated with desired doses of carnosol for 24 . Cell lysates were prepared and cleaved caspase-3 levels were evaluated by ELISA. Cleaved caspase- 3 levels for treated HCT116 and SW480 cells. These experiments are represented by the mean along with standard deviation compared to control cells, " $P<0.05$, ${ }^{* *} P<0.01$ 
[A]

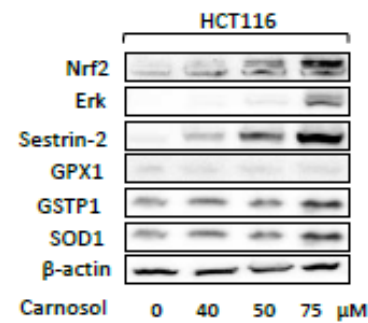

[B]
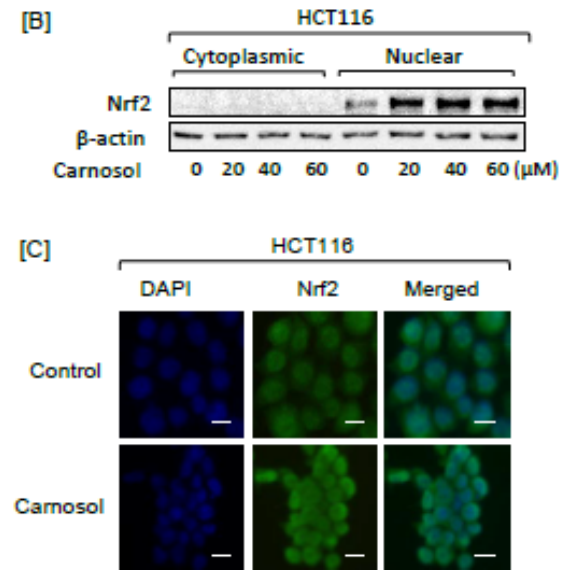
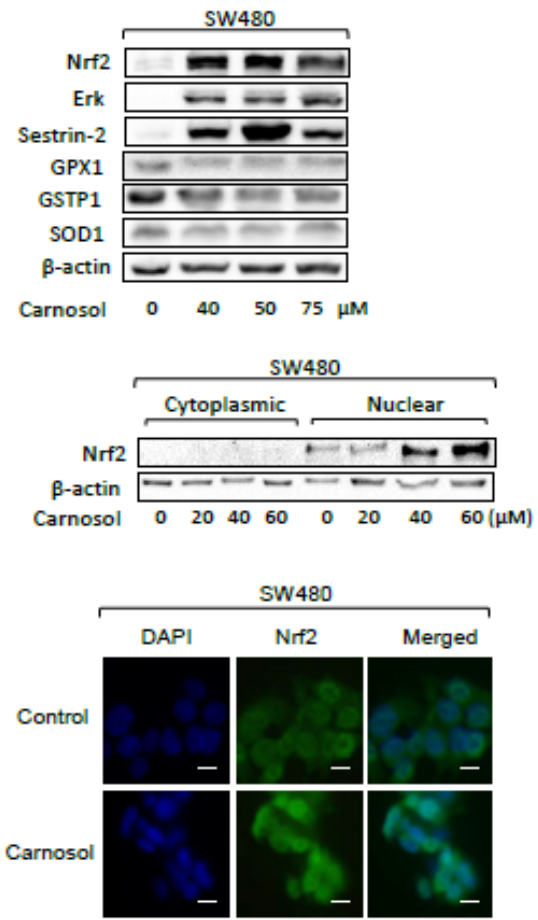

Figure 2. (A) Protein expression of selected proteins in carnosol-treated HCT116 and SW480 cells was performed after a 24-hour treatment. (B) Cytoplasmic and nuclear Nrf2 expression in carnosol-treated HCT116 and SW480 cells was determined by Western Blot following 24-hour treatment. (C) Expression of Nrf2 was visualized by immunofluorescence following a 24-hour treatment with carnosol in HCT116 and SW480 cells. Bar scale represents 50 px

PERK in colon cells. Increased expression of PERK and phospho-PERK was observed following treatment with carnosol in both HCT116 and SW480 cells (Figure 3A). Using immunofluorescence microscopy, up regulation of PERK and sestrin-2 protein expression can be visualized in HCT116 and SW480 cells (Figure 3B and 4A).

\section{Silencing of Nrf 2 by siRNA prevents carnosol induction of sestrin 2}

To further define the role of carnosol on the induction of Nrf2/ sestrin-2 signaling pathway, a more targeted inhibition of Nrf2 using siRNA was undertaken (Figure 4B). A combination of carnosol and Nrf2-siRNA led to a decreased expression of sestrin 2 in HCT116 cells compared to cells treated with isoliquiritigenin alone. These findings suggested that carnosol induces sestrin-2 expression by the activation of the Nrf2 pathway. Interestingly, complete silencing of sestrin 2 following Nrf2 siRNA treatment did not occur suggesting other mechanisms could be contributing to sestrin 2 expression (Figure 5).

\section{Discussion}

In summary, we observed carnosol to promote activation of the Nrf2 signaling network in two different cancer cell lines (i.e. HCT116 and SW480). An interesting observation is the possible link between activation of the unfolded protein response pathway and Nrf2. More specifically, Nrf2 has been shown to be a direct substrate for PERK by other investigators suggesting that a possible alternative mechanism different than the direct interaction with Keap1/Nrf2 complex can occur [18]. Phosphorylation of PERK will trigger disassociation of the Nrf2/Keap1 complex and inhibits their association [19]. This preliminary data provides an alternative explanation for activating $\mathrm{Nrf} 2$ in addition to other proposed mechanisms. One of the proposed mechanisms of carnosol is the conversion to a quinone species allowing for an electrophilic reaction with Nrf2/Keap1 machinery [21,22]. This mechanism is initiated by S-alkylation of the critical cysteine thiol of the Keap1 protein by the "electrophilic" quinone-type of carnosic acid or carnosol. Alternatively, carnosol through modulation of PERK could indirectly interact with the Keap1 proteins, thereby, releasing Nrf2. Previously, Valdez et al have shown that PERK can be modulated at the transcriptome level, however we provide evidence that carnosol promoted up regulation of PERK and increased phosphorylation of PERK in HCT116 and SW480 cells [23]. From our studies as well as other studies it is evident that carnosol interacts directly and indirectly with the Nrf2 machinery to regulate antioxidant gene expression (e.g. sestrin-2). Interestingly, silencing of Nrf2 did not seem to block the expression of sestrin 2 suggesting that alternative pathways should be explored for activation of sestrin-2. Shin et al. reported that the oxidative stress defense pathway Nrf2-ARE regulates induction of sestrin-2 expression [17]. Recently, Wei et al. reported that decreased expression of sestrin-2 predicts unfavorable outcome in colorectal cancer [24]. The implications of these results extend beyond cell culture and may further our understanding about the potential of carnosol and other compounds from rosemary (Rosmarinus officinalis) in promoting GI health.

The use of food preservatives is a billion-dollar industry with an increasing consumer demand for natural alternatives increasing. Natural food preservatives including rosemary extract are widely used in the EU and USA and may offer an unexpected benefit for gastrointestinal health. A perceived weakness could be the use of carnosol concentrations near $40 \mu \mathrm{M}$ when other studies have reported concentrations as low as $5 \mu \mathrm{M}$ modulating gene expression [25]. There is strong evidence suggesting that diterpenes are well absorbed with multiple publications reporting plasma levels of carnosic acid at 105 $\mu \mathrm{M}$ and $126 \mu \mathrm{M}$ respectively after oral administration of $90 \mathrm{mg} / \mathrm{kg}$ and $64.3 \mathrm{mg} / \mathrm{kg}$, respectively [26,27]. Another pharmacokinetic study 
[A]

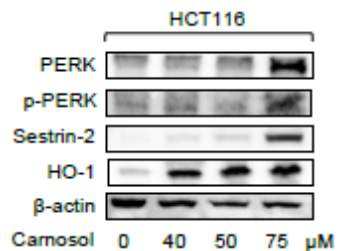

[B]

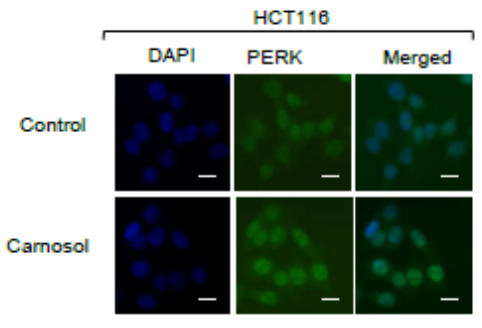

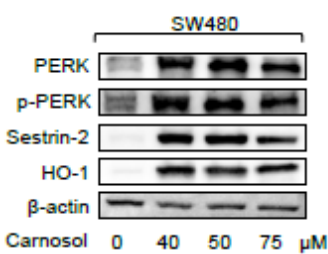

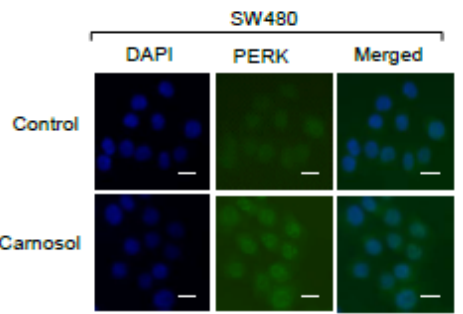

Figure 3. Carnosol promoted the expression of PERK, p-PERK, sestrin-2 and HO-1 in treated colon cancer cells. HCT116 and SW480 cells were treated with increasing doses of carnosol for $24 \mathrm{hr}$. Cell lysates were prepared and subjected to western blot for detecting the expression of ER stress proteins and antioxidant enzymes. (A) Expression of PERK, and phosphor-PERK, sestrin-2 and HO-1 was determined by Western blot. (B) Intracellular immunofluorescent staining of PERK in carnosol-treated HCT116 and SW480 cells. Left column, DAPI-stained nuclei appear blue; middle column: FITC-stained cells appear green showing PERK expression; right column: merged pictures of DAPI and FITC. Bar scale represents 50 px

[A]

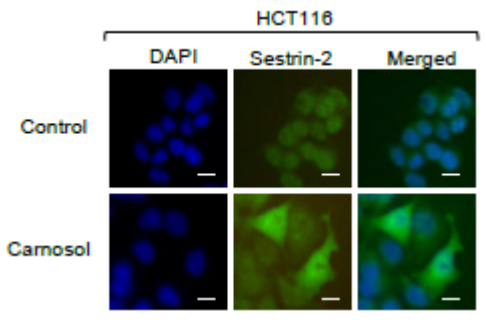

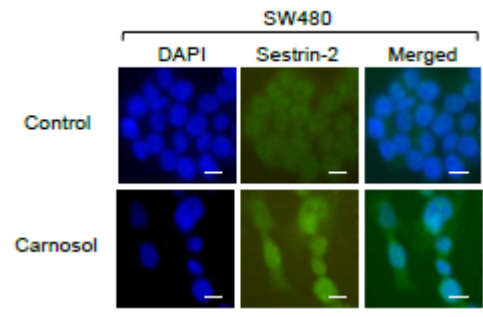

$[\mathrm{B}]$

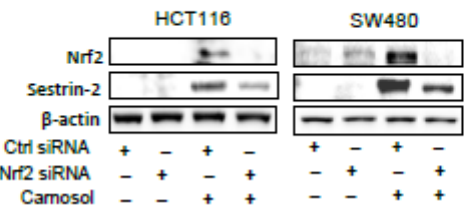

Figure 4. Carnosol modulate the Nrf2/Sestrin-2 pathway in colon cancer cells. (A) Intracellular immunofluorescent staining of sestrin-2 in carnosol-treated HCT116 and SW480 cells. For each panel: Left column, DAPI-stained nuclei appear blue; middle column: FITC-stained cells appear green showing sestrin-2 expression; right column: merged pictures of DAPI and FITC. Bar scale represents 50 px. (B) Cells were treated with Nrf2-siRNA with or without carnosol for $24 \mathrm{~h}$. Cell lysates were prepared and subjected to Western blot analysis. Minus and plus symbols represent absence and presence of indicated agents

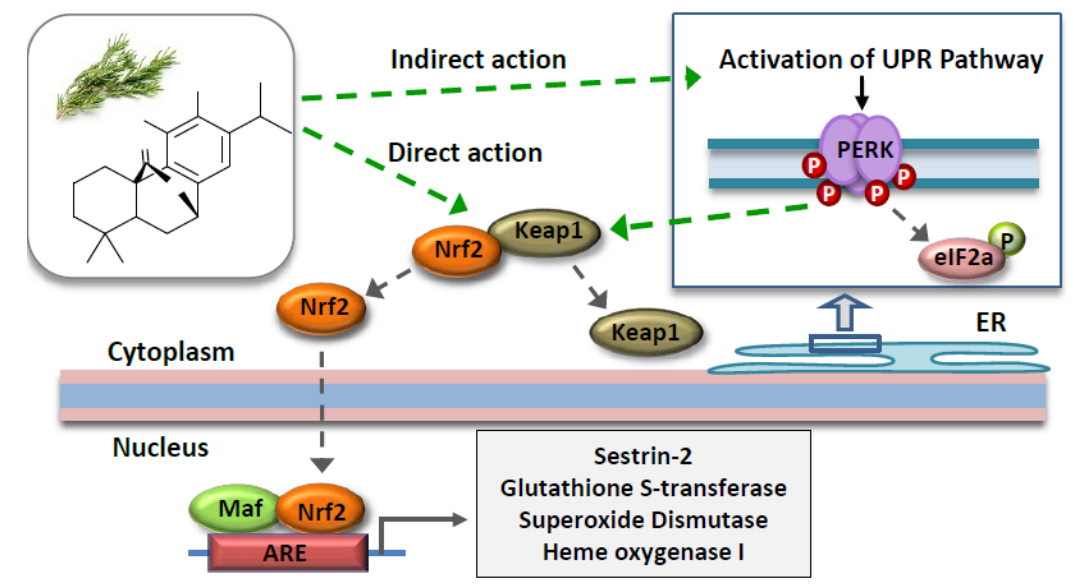

Figure 5. This figure depicts the action of carnosol from rosemary that will interact directly with Keap1/Nrf2 and releasing Keap1 from Nrf2. This action prevents the ubiquitination of Nrf2 and rapid degradation thereby accumulating and translocating to the nucleus to transcribe detoxification and antioxidant enzymes (e.g. Sestrin-2, GST, SOD, HO-1). Diterpenes similar to carnosol have also been reported to activate the UPR pathway leading to the phosphorylation of PERK that can act as a direct substrate for Nrf2 releasing Keap1 
reported on the administration of a rosemary extract that contained carnosol (i.e. equivalent dose of $15 \mathrm{mg} / \mathrm{kg}$ orally) to achieve plasma levels of $18.2 \mu \mathrm{M}$ [28]. Using the FDA guidance on dose scaling which we have described previously, a dose of $15 \mathrm{mg} / \mathrm{kg}$ in rats, would be the human equivalent dose of $146 \mathrm{mg}$ in a $60 \mathrm{~kg}$ human adult [29-31]. In the SW480 cell line phosphorylation of PERK and sestrin-2 expression was observed at $40 \mu \mathrm{M}$ and given the extent of activation likely could be achieved with lower concentrations of carnosol. Diterpenes have been shown to be well absorbed orally with bioavailability estimated to be as high as $60 \%[28,32]$. Further work is needed to understand the complexities that occur with scaling doses from animals such as rats to humans, however, our results in combination with pharmacokinetic results appear to be promising in understanding the potential benefits of natural antioxidant food preservatives for promoting gastrointestinal health through activation of the cellular antioxidant sestrin-2.

In conclusion, these results suggest that carnosol can increase Nrf2 activity leading to the induction of sestrin 2 . These results appear to be promising as there are few compounds that have been reported to activate sestrin 2 in colon cells. This is significant because low sestrin 2 expression is associated with specific colon cancers and represents a promosing strategy for improving gastrointestinal health.

\section{Acknowledgements}

Aspects of this work was supported by the National Institute of Food and Agriculture (Award Number \#: 2017-67017-26364, Proposal Number \#: 2016-09958, Accession \#: 1011859) to J.Johnson. Miao Yan was supported by the National Natural Science Foundation of China (No. 81202985) and China Scholarships Council (No. 201208430206).

\section{Disclosure statement}

The authors declare no conflict of interest.

\section{References}

1. de Lorgeril M (2013) Mediterranean diet and cardiovascular disease: Historical perspective and latest evidence. Curr Atheroscler Rep 15: 370. [Crossref]

2. Ngo SN, Williams DB, Head RJ (2011) Rosemary and cancer prevention: Preclinical perspectives. Crit Rev Food Sci Nutr 51: 946-954. [Crossref]

3. Petiwala SM, Johnson JJ (2015) Diterpenes from rosemary (rosmarinus officinalis): Defining their potential for anti-cancer activity. Cancer Lett 367: 93-102. [Crossref]

4. Petiwala SM, Puthenveetil AG, Johnson JJ (2013) Polyphenols from the Mediterranean herb rosemary (rosmarinus officinalis) for prostate cancer. Front Pharmacol 4: 29. [Crossref]

5. Gotsis E, Anagnostis P, Mariolis A, Vlachou A, Katsiki N, et al. (2015) Health benefits of the Mediterranean Diet: an update of research over the last 5 years. Angiology 66: 304-318. [Crossref]

6. Grosso G, Mistretta A, Frigiola A, Gruttadauria S, Biondi A, et al. (2014) Mediterranean diet and cardiovascular risk factors: a systematic review. Crit Rev Food Sci Nutr 54: 593-610. [Crossref]

7. Aguilar F, Autrup H, Barlow S, Castle L, Crebelli R, et al. (2008) Use of rosemary extracts as a food addtive - scientific opinion of the panel on food additives, flavourings, processing aids and materials in contact with food. The EFSA Journal 8: 1-29.

8. Lo AH, Liang YC, Lin-Shiau SY, Ho CT, Lin JK (2002) Carnosol, an antioxidant in rosemary, suppresses inducible nitric oxide synthase through down-regulating nuclear factor-kappab in mouse macrophages. Carcinogenesis 23: 983-991. [Crossref]

9. Aruoma OI, Halliwell B, Aeschbach R, Löligers J (1992) Antioxidant and pro-oxidant properties of active rosemary constituents: carnosol and carnosic acid. Xenobiotica 22: 257-268. [Crossref]
10. Munne-Bosch S, Alegre L (2001) Subcellular compartmentation of the diterpene carnosic acid and its derivatives in the leaves of rosemary. Plant Physiol 125: 10941102. [Crossref]

11. Johnson JJ, Syed DN, Heren CR, Suh Y, Adhami VM, et al. (2008) Carnosol, a dietary diterpene, displays growth inhibitory effects in human prostate cancer pc 3 cells leading to g2-phase cell cycle arrest and targets the 5'-amp-activated protein kinase (ampk) pathway. Pharm Res 25: 2125-2134. [Crossref]

12. Li G, Petiwala SM, Pierce DR, Nonn L, Johnson JJ (2013) Selective modulation of endoplasmic reticulum stress markers in prostate cancer cells by a standardized mangosteen fruit extract. PLoS One 8: e81572. [Crossref]

13. Johnson JJ, Syed DN, Suh Y, Heren CR, Saleem M, et al. (2010) Disruption of androgen and estrogen receptor activity in prostate cancer by a novel dietary diterpene carnosol: Implications for chemoprevention. Cancer Prev Res 3: 1112-1123. [Crossref]

14. Roberts RA, Smith RA, Safe S, Szabo C, Tjalkens RB, et al. (2010) Toxicologica and pathophysiological roles of reactive oxygen and nitrogen species. Toxicology 276 : 85-94. [Crossref]

15. Ma Q, He X (2012) Molecular basis of electrophilic and oxidative defense: promises and perils of Nrf2. Pharmacol Rev 64: 1055-1081. [Crossref]

16. Yan M, Li G, Petiwala SM, Householter E, Johnson JJ (2015) Standardized rosemary (rosmarinus officinalis) extract induces nrf2/sestrin-2 pathway in colon cancer cells. $J$ Funct Foods 13: 137-147.

17. Shin BY, Jin SH, Cho IJ, Ki SH (2012) Nrf2-ARE pathway regulates induction of Sestrin-2 expression. Free Radic Biol Med 53: 834-841. [Crossref]

18. Velichkova M, Hasson T (2005) Keap1 regulates the oxidation-sensitive shuttling of nrf2 into and out of the nucleus via a crm1-dependent nuclear export mechanism. Mol Cell Biol 25: 4501-4513. [Crossref]

19. Cullinan SB, Zhang D, Hannink M, Arvisais E, Kaufman RJ, et al. (2003) Nrf2 is a direct PERK substrate and effector of PERK-dependent cell survival. Mol Cell Biol 23: 7198-7209. [Crossref]

20. Petiwala SM, Berhe S, Li G, Puthenveetil AG, Rahman O, et al. (2014) Rosemary (rosmarinus officinalis) extract modulates chop/gadd153 to promote androgen receptor degradation and decreases xenograft tumor growth. PLoS One 9: e89772. [Crossref]

21. Satoh T, Izumi M, Inukai Y, Tsutsumi Y, Nakayama N, et al. (2008) Carnosic acid protects neuronal ht 22 cells through activation of the antioxidant-responsive element in free carboxylic acid- and catechol hydroxyl moieties-dependent manners. Neurosci Lett 434: 260-265. [Crossref]

22. Satoh T, Kosaka K, Itoh K, Kobayashi A, Yamamoto M, et al. (2008) Carnosic acid, a catechol-type electrophilic compound, protects neurons both in vitro and in vivo through activation of the keap1/nrf2 pathway via s-alkylation of targeted cysteines on keap1. J Neurochem 104: 1116-1131. [Crossref]

23. Valdes A, Garcia-Canas V, Rocamora-Reverte L, Gomez-Martinez A, Ferragut JA, et al. (2013) Effect of rosemary polyphenols on human colon cancer cells: Transcriptomic profiling and functional enrichment analysis. Genes Nutr 8: 43-60. [Crossref]

24. Wei JL, Fu ZX, Fang M, Guo JB, Zhao QN, et al. (2015) Decreased expression of sestrin 2 predicts unfavorable outcome in colorectal cancer. Oncol Rep 33: 1349-1357. [Crossref]

25. Chun KS, Kundu J, Chae IG, Kundu JK (2014) Carnosol: a phenolic diterpene with cancer chemopreventive potential. J Cancer Prev 19: 103-110. [Crossref]

26. Yan H, Wang L, Li X, Yu C, Zhang K, et al. (2009) High-performance liquid chromatography method for determination of carnosic acid in rat plasma and its application to pharmacokinetic study. Biomed Chromatogr 23: 776-781. [Crossref]

27. Doolaege EH, Raes K, De Vos F, Verhé R, De Smet S (2011) Absorption, distribution and elimination of carnosic acid, a natural antioxidant from Rosmarinus officinalis, in rats. Plant Foods Hum Nutr 66: 196-202. [Crossref]

28. Romo Vaquero M, Garcia Villalba R, Larrosa M, Yanez-Gascon MJ, Fromentin E, et al. (2013) Bioavailability of the major bioactive diterpenoids in a rosemary extract Metabolic profile in the intestine, liver, plasma, and brain of zucker rats. Mol Nutr Food Res 57: 1834-1846. [Crossref]

29. Ramaiya A, Li G, Petiwala SM, Johnson JJ (2012) Single dose oral pharmacokinetic profile of Î \pm -mangostin in mice. Curr Drug Targets 13: 1698-1704. [Crossref]

30. Petiwala SM, Li G, Ramaiya A, Kumar A, Johnson JJ (2014) Pharmacokinetic characterization of mangosteen (garcinia mangostana) fruit extract standardized to alpha-mangostin in c57bl/6 mice. Nutr Res 34: 336-345. [Crossref] 
Yan M (2018) Carnosol, a dietary diterpene from rosemary (Rosmarinus officinalis) activates Nrf2 leading to sestrin 2 induction in colon cells

31. Li G, Petiwala SM, Nonn L, Johnson JJ (2014) Inhibition of chop accentuates the apoptotic effect of alpha-mangostin from the mangosteen fruit (garcinia mangostana) in 22rv1 prostate cancer cells. Biochem Biophys Res Commun 453: 75-80. [Crossref]
32. Soler-Rivas C, Marin FR, Santoyo S, Garcia-Risco MR, Senorans FJ, et al. (2010) Testing and enhancing the in vitro bioaccessibility and bioavailability of rosmarinus officinalis extracts with a high level of antioxidant abietanes. J Agric Food Chem 58: 1144-1152. [Crossref]

Copyright: (C2018 Yan M. This is an open-access article distributed under the terms of the Creative Commons Attribution License, which permits unrestricted use, distribution, and reproduction in any medium, provided the original author and source are credited. 